JOURNAL OF THEORETICAL

AND APPLIED MECHANICS

53, 1 , pp. 69-80, Warsaw 2015

DOI: $10.15632 /$ jtam-pl.53.1.69

\title{
DYNAMICS OF AN UNGUIDED MISSILES LAUNCHER
}

\author{
Zbigniew Dziopa, Piotr Buda, Maciej Nyckowski, Rafa€ Pawlikowski \\ Kielce University of Technology, Faculty of Mechatronics and Machine Building, Kielce, Poland \\ e-mail: zdziopa@tu.kielce.pl
}

\begin{abstract}
The paper discussed the modelling, examination of the dynamic properties and stability of the WW-4 launcher of unguided short-range missiles NLPR-70. Remotely controlled armament module ZSMU-70, in the presented configuration, is designed to combat ground targets in form of light armoured vehicles. The purpose of this paper is to present a physical model, a mathematical model and computer simulation of launcher motion during launching and the first phase of flight of the missile. The virtual model is formulated in the vertical plane and verified by experimental studies. The theoretical model corresponds to experimental studies conducted at the military testing ground with application of a high-speed camera.
\end{abstract}

Keywords: dynamics, launcher, unguided missiles, virtual model, experimental studies, stabilization

\section{Introduction}

The paper presents the WW-4 launcher of short-range unguided missiles NLPR-70, as shown in Fig. 1.

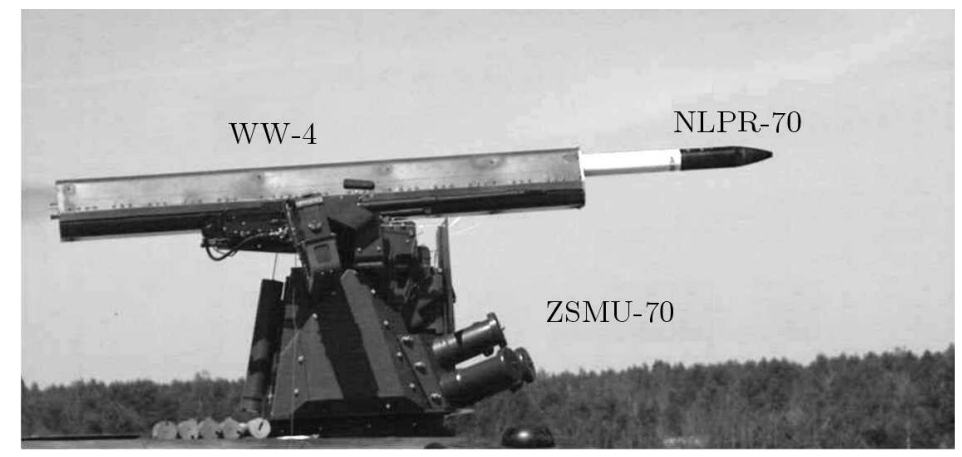

Fig. 1. The WW-4 launcher of unguided missiles NLPR-70

The ZSMU-70 remotely controlled weapon consists of a launcher mounted on an armoured motor vehicle (Dziopa, 2004, 2007). The launcher structure includes a platform with a guide mounted on it. The guide can rotate relative to the platform performing the process of proper angular setting selection. The unguided missile is located inside the guide. After the angular settings are selected, the guide stops and the missile is launched. The rocket motor of the missile, which generates thrust with known characteristics, is activated. A proper geometry of the rocket motor nozzle leads to a force distribution which causes motion of the missile along the launcher guide and at the same time its revolution round the longitudinal axis.

The presented system is designated to combat ground targets such as light armoured vehicles and military infrastructure. The launch of the NLPR-70 missile from the WW-4 launcher was recorded using a high-speed camera at the military testing ground. The vehicle does not perform translatory motion while launching the missile. Firing is conducted from a stationary vehicle. 
Figure 2 illustrates sample film frames which capture the first phase of the launcher motion. The phase is characterized by direct contact between the moving missile and the guide.

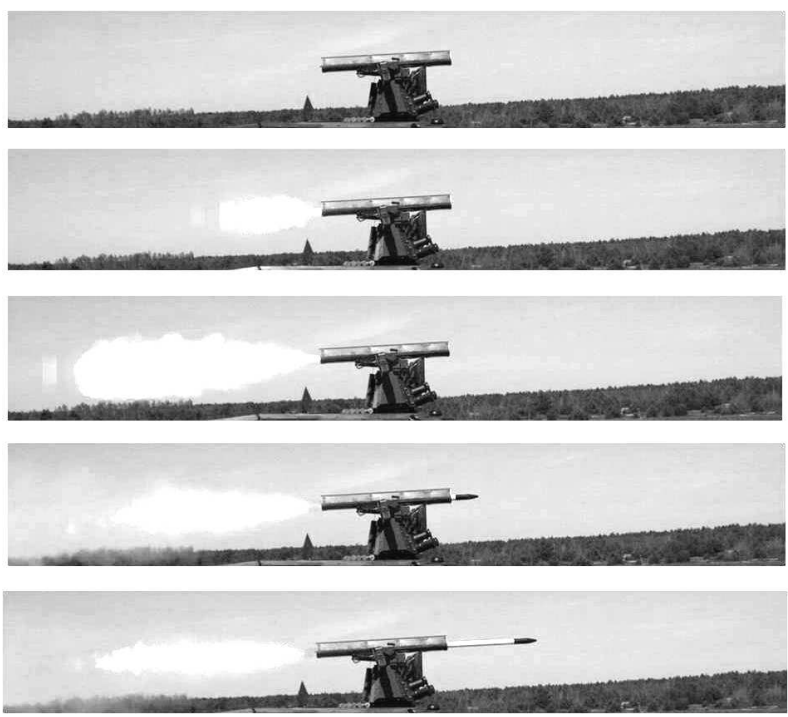

Fig. 2. The first phase of the launcher motion
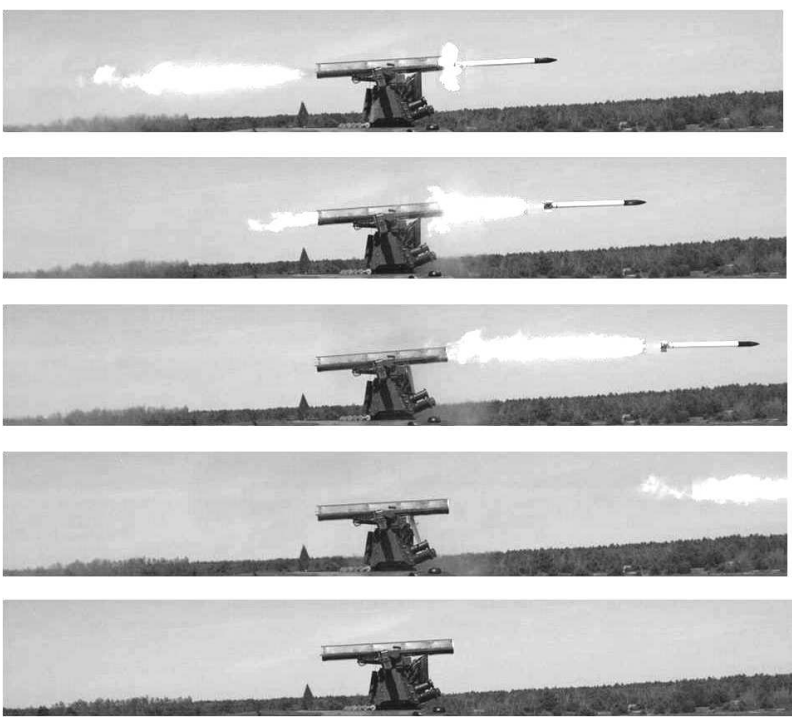

Fig. 3. The second and third phase of the launcher motion

The system is composed of two interacting objects: the launcher and the unguided rocket missile. The motion of the launcher is induced exclusively by the missile launch. The missile moves along the launcher guide, which results in a change of the characteristics of the system inertia. The change involves the mass, weight distribution and the location of the system mass centre (Biessonov, 1967). Figure 3 shows sample frames capturing the second and third phases of the launcher motion. At the second phase, the missile leaves the guide and begins the flight towards the target. The launcher stops being a system variable in time. At the moment the missile leaves the launcher, the system disintegrates into two objects: the launcher and the unguided rocket missile. They are not yet independent objects as the external input starts affecting the launcher. The input results from the influence of the rocket motor of the moving away missile. The input works until the rocket motor of the missile has no influence on motion of the launcher. It is only at the third phase that the launcher and unguided missile become two independent 
objects. Affected by the missile launch, the launcher vibration is excited. The missile performs the trajectory resulting from the initial flight parameters developed on the launcher.

The motion of the armament module recorded under field conditions demonstrated the possibility of simplifying the spatial model down to two dimensions. The studied system is characterized by asymmetry of both geometry and the inertia characteristics. The verification of the developed theoretical model has been conducted on the basis of the analysis of the picture recorded at the military testing ground. Therefore, the ZSMU-70 armament module can be subjected to thorough analysis with computer simulation. It is significant from the perspective of costs of the performed analyses, which are much higher in the case of studies at the testing ground. Equally important is the aspect of testing the launcher via the computer under extreme conditions which can be formulated by applying numerical methods.

\section{Physical model of the launcher}

The physical model, which is presented in Fig. 4 (Gantmacher, 1970; Osiecki, 1994; Swietlickij, 1963), has been developed on the basis of the WW-4 launcher of the NLPR-70 unguided missiles. The model presented in this figure illustrates the first phase of the launcher motion.

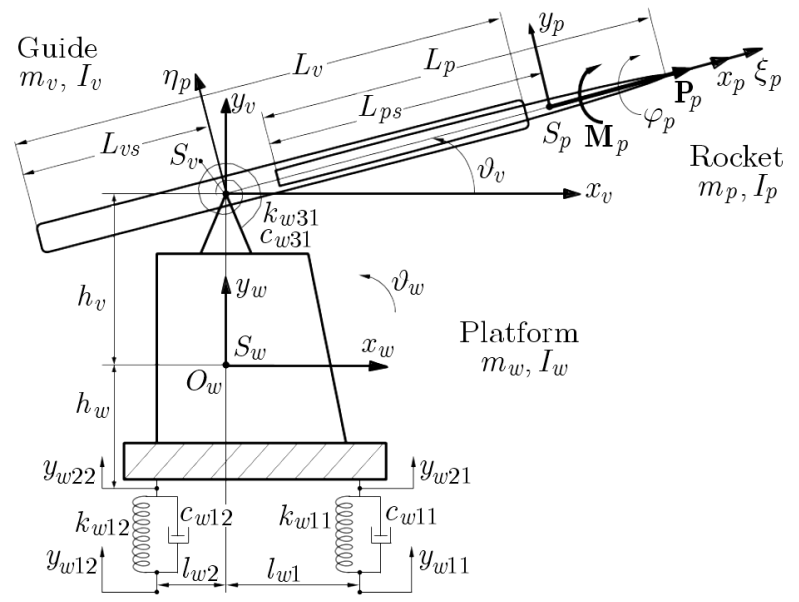

Fig. 4. Physical model of the missile launcher

The launcher, which is mounted on a motor vehicle, consists of two basic objects in form of a platform and a guide.It ias assumed that the launcher along with the guide is positioned on the vehicle symmetrically towards the vertical plane passing through the centre of mass of the vehicle. The launcher is modelled in form of two masses and three deformable elements, as in Fig. 4. The platform is a perfectly rigid body with mass $m_{w}$ and inertia moment $I_{w}$. The platform is mounted on the vehicle body with two passive spring-damping elements with linear parameters $k_{w 11}$ and $c_{w 11}$, and $k_{w 12}$ and $c_{w 12}$, respectively. The guide which is a perfectly rigid body with mass $m_{v}$ and inertia moment $I_{v}$ is mounted on the platform.

There is a rotational link between the guide and the platform. A deformable element with linear parameters $k_{w 31}$ and $c_{w 31}$ respectively ias modelled at the place of attachment. Figure 4 presents also geometrical characteristics of the launcher, which is necessary for conductingan analysis of the assembly dynamics.

The location of the solid body with mass $m_{w}$ and inertia moment $I_{w}$ and the body of the guide with mass $m_{v}$ and inertia moment $I_{v}$ at any point in time are determined in Cartesian dextrose rectangular coordinates system. The following coordinates are the reference system:

a) coordinates defining motion of the platform: $0_{w} x_{w} y_{w} z_{w}, S_{w} x_{w} y_{w} z_{w}, S_{w} \xi_{w} \eta_{w} \zeta_{w}$,

b) coordinates defining mption of the guide: $0_{v} x_{v} y_{v} z_{v}, S_{v} x_{v} y_{v} z_{v}, S_{v} \xi_{p} \eta_{p} \zeta_{p}$, 
The number of degrees of freedom resulting from the formulated structure of the launcher model describing the disorders of the primary motion in the vertical plane equals 3 . Two independent generalized coordinates are adopted in order to determine the position of the platform with mass $m_{w}$ and inertia moment $I_{w}$ at any moment in time: $y_{w}$ - vertical relocation of the centre of mass $S_{w}$ of the platform, $\vartheta_{w}$ - revolution angle of the platform round the axis $S_{w} z_{w}$.

One independent generalized coordinate has been adopted to determine the positions of the guide with mass $m_{v}$ and inertia moment $I_{v}$ at any moment in time: $\vartheta_{v}$ - rotation angle of the guide round the axis $S_{v} z_{v}$.

In the general case, the missile is an object with variable mass and weight distribution (Biessonov, 1967). Missile parameters such as mass $m_{p}$ and the tensor of moment of inertia $\widehat{I}_{p}$ are functions variable in time in the general case. The positions of the solid body at any moment in time are indicated by Cartesian orthogonal dextrose systems of rectangular coordinates. The following systems of coordinates constitute the systems of reference.

The systems of coordinates defining motion of the missile at the launch from the guide:

a) the system of coordinates defining the lifting motion of the missile:

- $0 x y z, 0_{v} x_{v} y_{v} z_{v}, S_{v} x_{v} y_{v} z_{v}$ and $S_{v} \xi_{p} \eta_{p} \zeta_{p}$ are systems of coordinates which have been discussed earlier, define one motion of the guide. The guide imposes constraints on the missile motion. The missile performs complex motion consisting of lifting motion performed by the guide and motion of the missile relative to the guide. In the case of launching the missile from the guide the system of coordinates $0 x y z, 0_{v} x_{v} y_{v} z_{v}$, $S_{v} x_{v} y_{v} z_{v}$ and $S_{v} \xi_{p} \eta_{p} \zeta_{p}$ define also the lifting motion of the missile;

b) the systems of coordinates defining the relative motion of the missile:

$-S_{p} \xi_{p} \eta_{p} \zeta_{p}$ is a system of coordinates performing linear motion relative to the system of coordinates $S_{v} \xi_{v} \eta_{v} \zeta_{v}$. The beginning of the system of coordinates $S_{p}$ at any moment in time coincides with the centre of mass. The condition of parallelism is always fulfilled for the axes $S_{p} \xi_{p}\left\|S_{v} \xi_{p}, S_{p} \eta_{p}\right\| S_{v} \eta_{p}$ and $S_{p} \zeta_{p} \| S_{v} \zeta_{p}$, corresponding one to another. Under the influence of the rocket booster, the missile centre of mass $S_{p}$ shifts along the axis $S_{v} \xi_{p}$

- $S_{p} x_{p} y_{p} z_{p}$ is a system of coordinates performing rotary motion relative to coordinates $S_{p} \xi_{p} \eta_{p} \zeta_{p}$. The axes $S_{p} x_{p}, S_{p} y_{p}$ and $S_{p} z_{p}$ are rigidly linked to the body of the missile in such a way that they become its main central inertia axes. The condition of parallelism is always fulfilled for the two axes $S_{p} \xi_{p} \| S_{p} x_{p}$. Under the influence of the booster, the body of the missile turns around axis $\mathrm{c}$ in accordance with the change of the tilting angle $\varphi_{p}$.

The number of degrees of freedom resulting from the formulated structure for the model of the kinematic pair: guide-missile which describes motion of the missile relative to the guide in the vertical plane equals 2 .

Two independent generalized coordinates are adopted in order to determine the position of the missile with mass $m_{p}$ and inertia moments $I_{p x p}, I_{p z p}$ at any moment in time: $\xi_{p}$ - linear relocation of the centre of mass $S_{p}$ of the missile along the axis $S_{v} \xi_{p}, \varphi_{p}$-rotation angle of the missile round the axis $S_{p} \xi_{p}$.

\section{Mathematical model of the launcher}

The mathematical model of the launcher in the vertical plane was developed on the basis of the adopted physical model (Gacek and Maryniak, 1987; Swietlickij, 1963). The discussed system was brought to the form of a structural model of discrete structure, therefore it is described 
by differential equations with ordinary derivatives represented by five independent generalized coordinates (De Silva, 2007; Gantmacher, 1970).

Five independent coordinates were adopted to determine the motion of the launcher model:

- platform - perfectly rigid body: $y_{w}, \vartheta_{w}$

- guide - perfectly rigid body: $\vartheta_{v}$

- missile - system variable in time: $\xi_{p}, \varphi_{p}$.

These are the equations of motion

$$
\begin{aligned}
& \left(m_{w}+m_{v}+m_{p}\right) \ddot{y}_{w}-\left(m_{v}+m_{p}\right) h_{v} \ddot{\vartheta}_{w} \sin \vartheta_{w}+m_{p} \xi_{p} \ddot{\vartheta}_{v} \cos \vartheta_{v} \\
& \quad-\left(m_{v}+m_{p}\right) h_{v} \dot{\vartheta}_{w}^{2} \cos \vartheta_{w}-m_{p} \xi_{p} \dot{\vartheta}_{v}^{2} \sin \vartheta_{v}+m_{p} \dot{\xi}_{p} \dot{\vartheta}_{v} \cos \vartheta_{v}+k_{w_{11}} \lambda_{w_{11}} \\
& \quad+k_{w_{12}} \lambda_{w_{12}}-c_{w_{11}} \dot{\lambda}_{w_{11}}-c_{w_{12}} \dot{\lambda}_{w_{12}}=-\left(m_{w}+m_{v}+m_{p}\right) g \\
& {\left[I_{w}+I_{p_{z p}}+I_{v}+\left(m_{v}+m_{p}\right) h_{v}^{2}\right] \ddot{\vartheta}_{w}+\left[I_{p_{z p}}+m_{p} h_{v} \xi_{p}\left(\cos \vartheta_{w} \sin \vartheta_{v}-\sin \vartheta_{w} \cos \vartheta_{v}\right)\right] \ddot{\vartheta}_{v}} \\
& \quad-\left(m_{v}+m_{p}\right) h_{v} \ddot{y}_{w} \sin \vartheta_{w}-m_{p} h_{v} \ddot{\xi}_{p}\left(\cos \vartheta_{w} \cos \vartheta_{v}+\sin \vartheta_{w} \sin \vartheta_{v}\right) \\
& \quad+2 m_{p} h_{v} \dot{\xi}_{p} \dot{\vartheta}_{w} \sin \vartheta_{w} \cos \vartheta_{v}-2 m_{p} h_{v} \dot{\xi}_{p} \dot{\vartheta}_{v} \sin \vartheta_{w} \cos \vartheta_{v} \\
& \quad+m_{p} h_{v} \xi_{p} \dot{\vartheta}_{v}^{2}\left(\cos \vartheta_{w} \cos \vartheta_{v}-\sin \vartheta_{w} \sin \vartheta_{v}\right)+k_{w_{11}} l_{w_{1}} \lambda_{w_{11}}-k_{w_{12}} l_{w_{2}} \lambda_{w_{12}} \\
& \quad-k_{w_{31}} \lambda_{w_{31}}-c_{w_{11}} l_{w_{1}} \dot{\lambda}_{w_{11}}+c_{w_{12}} l_{w_{2}} \dot{\lambda}_{w_{12}}+c_{w_{31}} \ddot{\lambda}_{w_{31}} \\
& \quad=\left(m_{v}+m_{p}\right) g h_{v} \sin \left(\vartheta_{w}+\vartheta_{w s t}\right) \\
& \left.\quad+I_{v}+I_{p_{z p}}+m_{p} \xi_{p}^{2}\right) \ddot{\vartheta}_{v}+m_{p} \xi_{p} \ddot{y}_{w} \cos \vartheta_{v} \\
& \left.\quad+I_{v}+I_{p_{z p}}+m_{p} h_{v} \xi_{p}\left(\cos \vartheta_{w} \sin \vartheta_{v}-\sin \vartheta_{w} \cos \vartheta_{v}\right)\right] \ddot{\vartheta}_{w}+2 m_{p} \xi_{p} \dot{\xi}_{p} \dot{\vartheta}_{v} \\
& \quad+m_{p} h_{v} \xi_{p} \dot{\vartheta}_{w}^{2}\left(\sin \vartheta_{w} \sin \vartheta_{v}+\cos \vartheta_{w} \cos \vartheta_{v}\right)+k_{w_{31}} \lambda_{w_{31}}-c_{w_{31}} \dot{\lambda}_{w_{31}} \\
& \quad=-m_{p} g \xi_{p} \cos \left(\vartheta_{v}+\vartheta_{v s t}\right) \\
& m_{p} \ddot{\xi}_{p}-m_{p} h_{v} \ddot{\vartheta}_{p}\left(\cos \vartheta_{w} \cos \vartheta_{v}+\sin \vartheta_{w} \sin \vartheta_{v}\right)+m_{p} \ddot{y}_{w} \sin \vartheta_{w} \\
& \quad+m_{p} h_{v} \dot{\vartheta}_{w}^{2}\left(\sin \vartheta_{w} \cos \vartheta_{v}-\cos \vartheta_{w} \sin \vartheta_{v}\right)+m_{p} \xi_{p} \dot{\vartheta}_{v}^{2}=-m_{p} g \sin \left(\vartheta_{v}+\vartheta_{v s t}\right)+P_{p} \\
& I_{p_{x p}} \ddot{\varphi}_{p}=M_{p}
\end{aligned}
$$

where

$$
\begin{aligned}
& \lambda_{w_{11}}=y_{w}+y_{w s t}+l_{w_{1}}\left(\vartheta_{w}+\vartheta_{w s t}\right)-y_{w_{11}} \\
& \lambda_{w_{12}}=y_{w}+y_{w s t}-l_{w_{2}}\left(\vartheta_{w}+\vartheta_{w s t}\right)-y_{w_{12}} \\
& \lambda_{w_{31}}=\vartheta_{v}+\vartheta_{v s t}-\vartheta_{w}+\vartheta_{w s t}
\end{aligned}
$$$$
\begin{aligned}
& \dot{\lambda}_{w_{11}}=y_{w}+l_{w_{1}} \dot{\vartheta}_{w}-y_{w_{11}} \\
& \dot{\lambda}_{w_{12}}=\dot{y}_{w}-l_{w_{2}} \dot{\vartheta}_{w}-\dot{y}_{w_{12}} \\
& \dot{\lambda}_{w_{31}}=\dot{\vartheta}_{v}-\dot{\vartheta}_{w}
\end{aligned}
$$

and $P_{p}$ - thrust of the rocket motor, $M_{p}$ - moment of force, generated by the rocket motor.

Three static displacements appear in the equations of motion for the launcher in the vertical plane:

- platform: $y_{w s t}, \vartheta_{w s t}$

- guide: $\vartheta_{v s t}$.

These are the static equilibrium equations

$$
\begin{aligned}
& k_{w_{11}}\left(y_{w s t}+l_{w_{1}} \vartheta_{w s t}\right)+k_{w_{12}}\left(y_{w s t}-l_{w_{2}} \vartheta_{w s t}\right)+\left(m_{w}+m_{v}+m_{p}\right) g=0 \\
& k_{w_{11}} l_{w_{1}}\left(y_{w s t}+l_{w_{1}} \vartheta_{w s t}\right)-k_{w_{12}} l_{w_{2}}\left(y_{w s t}-l_{w_{2}} \vartheta_{w s t}\right)-k_{w_{13}}\left(\vartheta_{v s t}+\vartheta_{w s t}\right) \\
& \quad-\left(m_{v}+m_{p}\right) g h_{v} \sin \vartheta_{w s t}=0 \\
& k_{w_{13}}\left(\vartheta_{v s t}+\vartheta_{w s t}\right)=0
\end{aligned}
$$

The mathematical model can be classified as a coupled system with time-varying coefficients, determined, variable in time, dissipative and bounded, with five degrees of freedom. This model corresponds to experimental studies. 


\section{Vibro-isolation system for controlling vibrations of the launcher}

The purpose of this paper is to present the vibro-isolation system reducing the occurring vibrations. Two methods for eliminating vibrations have been applied in the process of computer simulation (Engel and Kowal, 1995; Inman, 2006). Passive vibro-isolation is the first method, while the second is the hybrid stabilization method (Dziopa, 2003, 2008). The rheological VoigtKelvin model, which has been parametrically modified, is used in the passive method. The hybrid method uses control devices engaged in series in the weightless system with restitutional and dissipative properties. The applied control systems operate independently of each other. Each of them stabilizes only one point of attachment of the passive system. The adjustment takes place in a closed system(Inman, 2006). A passive element in the form of the Voight-Kelvin model along with the controlled executive system as an electro-hydraulic servo, two acceleration sensors and a numerical processor make the control loop. Using a double integrator, the measuring system transmits the realized signal and the forcing signal to the numerical processor, which on their basis indicates the control signal. The formulation of the signal through the numerical processor runs in accordance with the adopted control algorithm. The applied control systems provide both linear and angular stabilization of the launcher (Dziopa, 2003, 2008). The diagram of the control system for the US11 device is presented in Fig. 5.

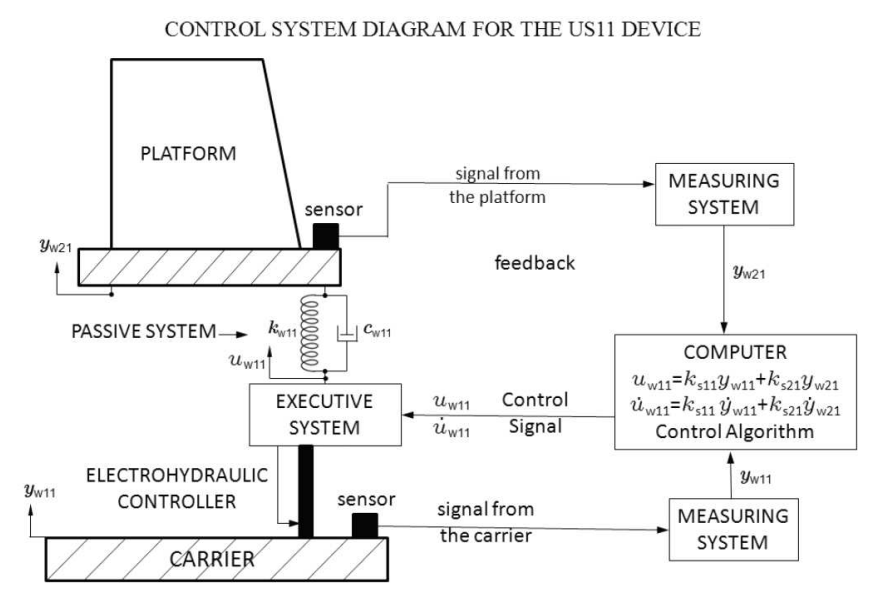

Fig. 5. Control system diagram for the US11 device

The adopted control algorithm realized in the process of simulation for the US11 device has the following form

$$
u_{w_{11}}=k_{s_{11}} y_{w_{11}}+k_{s_{21}} y_{w_{21}} \quad \dot{u}_{w_{11}}=k_{s_{11}} \dot{y}_{w_{11}}+k_{s_{21}} \dot{y}_{w_{21}}
$$

For the device US12

$$
u_{w_{12}}=k_{s_{12}} y_{w_{12}}+k_{s_{22}} y_{w_{22}} \quad \dot{u}_{w_{12}}=k_{s_{12}} \dot{y}_{w_{12}}+k_{s_{22}} \dot{y}_{w_{22}}
$$

where: $y_{w_{11}}, y_{w_{12}}$ - signals from the carrier, $y_{w_{21}}, y_{w_{22}}$ - signals from the platform, $k_{s_{11}}, k_{s_{12}}$, $k_{s_{21}}, k_{s_{22}}$ - control coefficients.

\section{Flight model missile}

The rocket missiles play an important part in the operation of the armament module ZSMU-70. Whether the object of attack will be neutralized depends directly on the missile. Effective firing of the unguided short-range rocket missile requires providing it with proper comfort. The missile 
may be excited to vibrate directly by the launcher which imposes constraints on the location and velocity of their points. In that case launcher vibrations may lead to failure in hitting the target with the missile already before it is launched (Dziopa, 2004). The launcher is one of the objects of the whole assembly and the disturbance it generates result from the interaction, which is the consequence of feedback within the system. The movement of the missile on the launcher is conditioned by the structure guide-missile. The rocket motor, whose function is to impart proper linear and angular velocity to the missile, is located at the rear end of the missile body. Therefore, on the launcher, the missile moves along the guide and rotates simultaneously round its own longitudinal axis (Gacek and Maryniak, 1987). After launching, the missile continues its movement towards the target. Although the launcher has no direct possibility to affect the missile during its flight, the interference in this phase of the flight takes place at the moment the missile leaves the guide. In one moment of time the system disintegrates in a natural way, i.e. it is divided into two independent systems in the form of the launcher and missile. At this moment, the initial kinematic parameters of the rocket flight are determined(Dziopa, 2006; Dziopa et al., 2010). The flight path is shaped, inter alia, by the value of these parameters. The launcher motion may cause the rocket to follow an adverse trajectory and consequently to miss the target.The missile moves in the gravitational field and in the Earth atmosphere (Żyluk and Pietraszek, 2014; Żyluk, 2014). The adopted model of forces affecting the rocket is presented in Fig. 6.

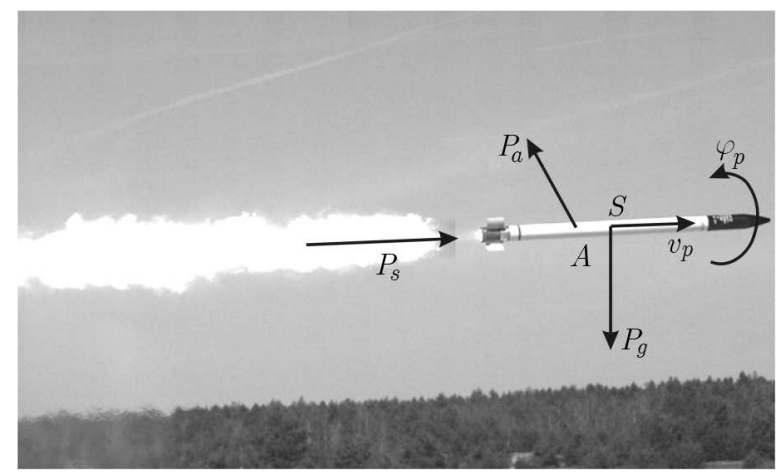

Fig. 6. The model of forces affecting the missile during its flight

Due to comlexity of the mathematical model of the rocket missile, only the dependencies representing the equations of motion are presented in the general form. The individual parameters appearing in the equations of motion are either described by functions or constitute empirical dependencies which are approximated in the process of numerical simulation.

The equations of missile motion in space are following (Dziopa, 2006; Dziopa et al., 2010; Sibilski, 2004):

- equations of the progressive part of motion in the system of coordinates connected to the flow $S_{p} x_{v} y_{v} z_{v}$

$$
\begin{aligned}
& m_{p} V_{p}=P_{s}\left[\sin \psi_{p} \sin \chi_{p}+\cos \psi_{p} \cos \chi_{p} \cos \left(\theta_{p}-\gamma_{p}\right)\right]-P_{g} \cos \chi_{p} \sin \gamma_{p} \\
& \quad+P_{a x}\left[\sin \psi_{p} \sin \chi_{p}+\cos \psi_{p} \cos \chi_{p} \cos \left(\theta_{p}-\gamma_{p}\right)\right] \\
& \quad+P_{a y}\left\{\sin \varphi_{p}\left[\sin \psi_{p} \cos \chi_{p} \cos \left(\theta_{p}-\gamma_{p}\right)-\cos \psi_{p} \sin \chi_{p}\right]-\cos \varphi_{p} \cos \chi_{p} \sin \left(\theta_{p}-\gamma_{p}\right)\right\} \\
& \quad+P_{a z}\left\{\sin \varphi_{p}\left[\sin \psi_{p} \cos \chi_{p} \cos \left(\theta_{p}-\gamma_{p}\right)-\cos \psi_{p} \sin \chi_{p}\right]-\cos \varphi_{p} \cos \chi_{p} \sin \left(\theta_{p}-\gamma_{p}\right)\right\} \\
& m_{p} V_{p} \dot{\gamma}_{p} \cos \chi_{p}=P_{s} \cos \psi_{p} \sin \left(\theta_{p}-\gamma_{p}\right)+P_{g} \cos \chi_{p}+P_{a x} \cos \psi_{p} \sin \left(\theta_{p}-\gamma_{p}\right) \\
& \quad+P_{a y}\left[\sin \varphi_{p} \sin \psi_{p} \sin \left(\theta_{p}-\gamma_{p}\right)-\cos \varphi_{p} \sin \left(\theta_{p}-\gamma_{p}\right)\right] \\
& \quad+P_{a z}\left[\sin \varphi_{p} \sin \psi_{p} \sin \left(\theta_{p}-\gamma_{p}\right)-\cos \varphi_{p} \sin \left(\theta_{p}-\gamma_{p}\right)\right]
\end{aligned}
$$




$$
\begin{aligned}
& m_{p} \dot{V}_{p} \dot{\gamma}_{p} \cos \chi_{p}=P_{s}\left[\cos \psi_{p} \cos \chi_{p} \cos \left(\theta_{p}-\gamma_{p}\right)-\sin \psi_{p} \cos \chi_{p}\right]-P_{g} \sin \chi_{p} \sin \gamma_{p} \\
& \quad+P_{a x}\left[\cos \psi_{p} \sin \chi_{p} \cos \left(\theta_{p}-\gamma_{p}\right)-\sin \psi_{p} \cos \chi_{p}\right] \\
& \quad+P_{a y}\left\{\sin \varphi_{p}\left[\sin \psi_{p} \cos \chi_{p} \cos \left(\theta_{p}-\gamma_{p}\right)+\cos \psi_{p} \sin \chi_{p}\right]-\cos \varphi_{p} \sin \chi_{p} \sin \left(\theta_{p}-\gamma_{p}\right)\right\} \\
& \quad+P_{a z}\left\{\sin \varphi_{p}\left[\sin \psi_{p} \sin \chi_{p} \cos \left(\theta_{p}-\gamma_{p}\right)+\cos \psi_{p} \cos \chi_{p}\right]-\sin \varphi_{p} \sin \chi_{p} \sin \left(\theta_{p}-\gamma_{p}\right)\right\}
\end{aligned}
$$

- equations of the spherical part of motion in the system of coordinates $S_{p} x_{p} y_{p} z_{p}$ related to the rocket

$$
\begin{aligned}
& I_{p x} \dot{\omega}_{p x}+\left(I_{p z}-I_{p y}\right) \omega_{p y} \omega_{p z}=M_{a x} \quad I_{p y} \dot{\omega}_{p y}+\left(I_{p x}-I_{p z}\right) \omega_{p x} \omega_{p z}=M_{a y} \\
& I_{p z} \dot{\omega}_{p z}+\left(I_{p y}-I_{p x}\right) \omega_{p x} \omega_{p y}=M_{a z}
\end{aligned}
$$

where: $V_{p}$ - velocity of the rocket mass centre, $\gamma_{p}, \chi_{p}$ - inclination angle of the vector of deviation of the missile velocity, $\theta_{p}, \psi_{p}, \varphi_{p}$ - inclination angle, deviation, tilt of the rocket body, $\omega_{p x}, \omega_{p y}, \omega_{p z}$ - components of the vector of missile angular velocity, $m_{p}$ - rocket mass, $I_{p x}, I_{p y}, I_{p z}$ - principal central moments of inertia, $P_{a x}, P_{a y}, P_{a z}$ - aerodynamic force vector components, $M_{a x}, M_{a y}, M_{a z}$ - vector components of the aerodynamic moment, $P_{g}$ - force of gravity, $P_{s}$ - rocket motor thrust.

\section{Numerical simulation}

The formulated model of the missile launcher allowed us to conduct numerical simulation aiming to analyze the launcher motion while launching the missile and during its flight (Dziopa, 2004, 2006; Dziopa et al., 2010). The present configuration of the launcher is dependent on angular settings resulting from the location of the target. During the launch of the missile, the launcher is a system variable in time (Biessonov, 1967). Its mass as well as its weight distribution change. Sample results of the conducted numerical simulation are presented below.

Figure 7 presents variability courses of the angle of inclination of the launcher guide.

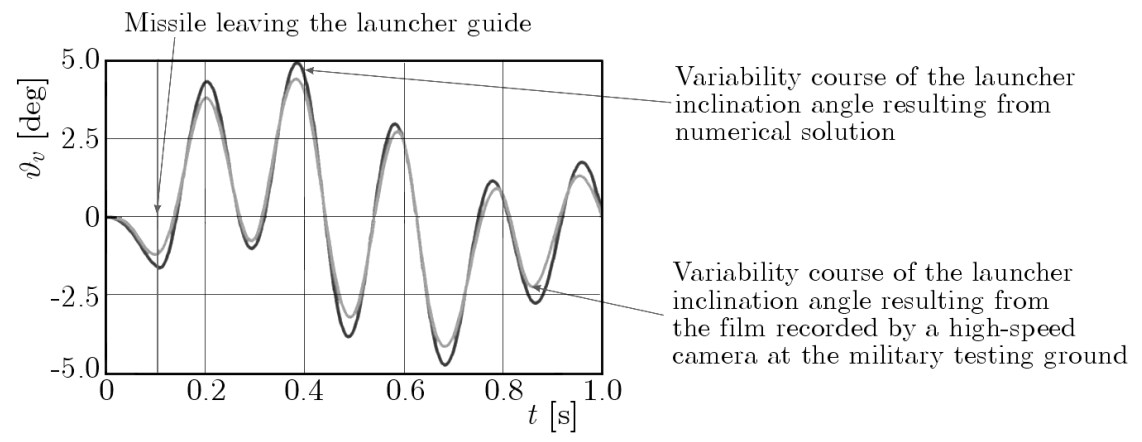

Fig. 7. Variability course of the launcher guide inclination angle

Figures 8 present variability courses of the displacementin of the launcher platform in the vertical motion.

Figure 9 shows the variability course of the inclination angle of the guide in the case of active and passive mounting of the platform. Within the whole range of variability of functions, the hybrid vibro-isloation system leads to a significant reduction of angular vibrations of the guide. The application of the passive system only does not provide sufficient vibro-isolation.

Figure 10 illustrates a comparison of the linear dislocation of the platform in the case of active and passive mounting. Within the entire scope of the function variability, the hybrid system of vibro-isolation entails definite reduction of linear vibrations of the platform. The application of the passive system exclusively does not provide sufficient vibro-isolation. 


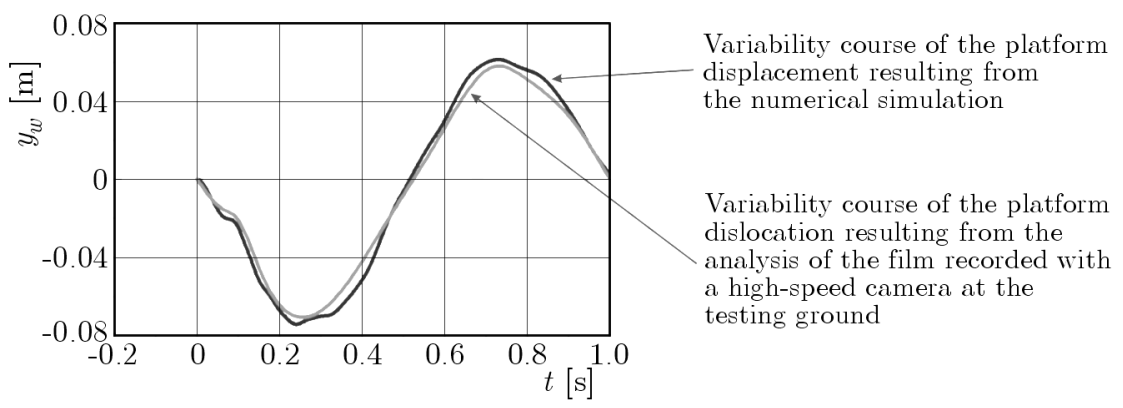

Fig. 8. The course of variability of launcher platform dislocation

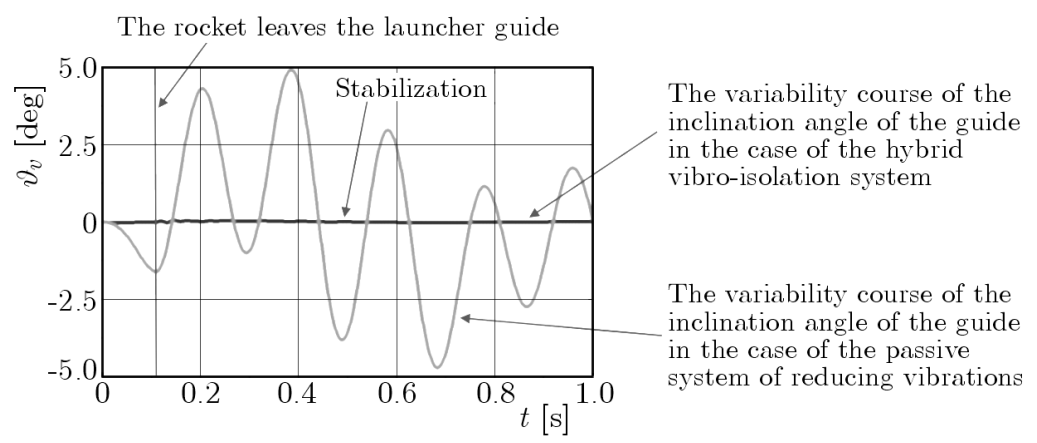

Fig. 9. Comparison of the variability course of the inclination angle of the platform in the case of its active and passive mounting

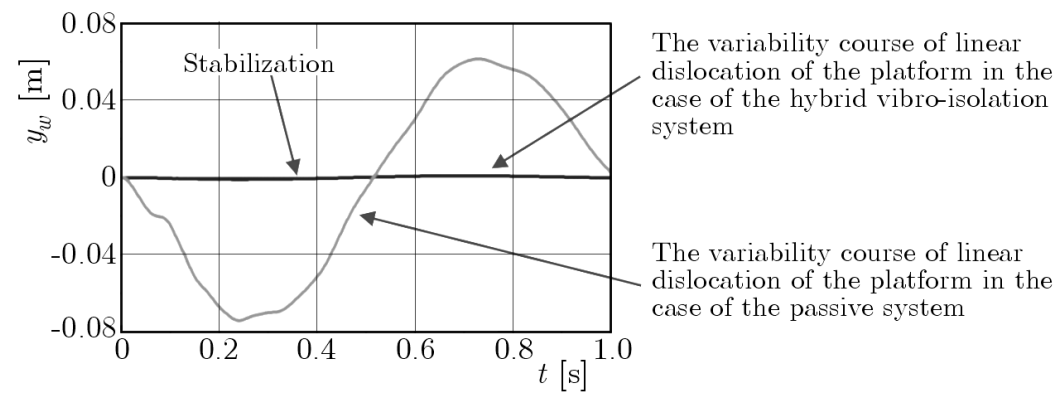

Fig. 10. Comparison of the linear dislocation of the platform in the case of its active and passive mounting

Sample results of the numerical simulation of flight of the unguided short-range rocket missile are presented below. After launching, the rocket starts its flight having various initial parameters. The quantitative and qualitative diversification of the initial parameters results from the dynamic reaction appearing in the launcher system from which the missile is fired. One of the initial parameters is the inclination angle of the rocket $\theta_{p}$ determined at the moment of launching. The results include three values of the initial inclination angle of the rocket: $1-\theta_{p}=10 \mathrm{deg}$, $2-\theta_{p}=8 \mathrm{deg}, 3-\theta_{p}=6 \mathrm{deg}$.

A slight change in the inclination angle of the rocket missile at the moment when the missile leaves the launcher guide significantly changes the flight trajectory, as in Fig. 11. Missiles 2 and 3 realize a wrong trajectory and, as a result, miss the target. The courses of variability of the angular velocity in the tilt motion of missiles 1, 2, and 3 are the same, see Fig. 11.

It results from the conducted research that it is the angular velocity in the inclination movement $\dot{\theta}_{p}$ that has the most significant influence on the performance of the rocket during its flight. It is the angular velocity defined at the moment the missile leaves the launcher guide. The flight path of the rocket depends not only on the value of this kinematic quantity, but also 

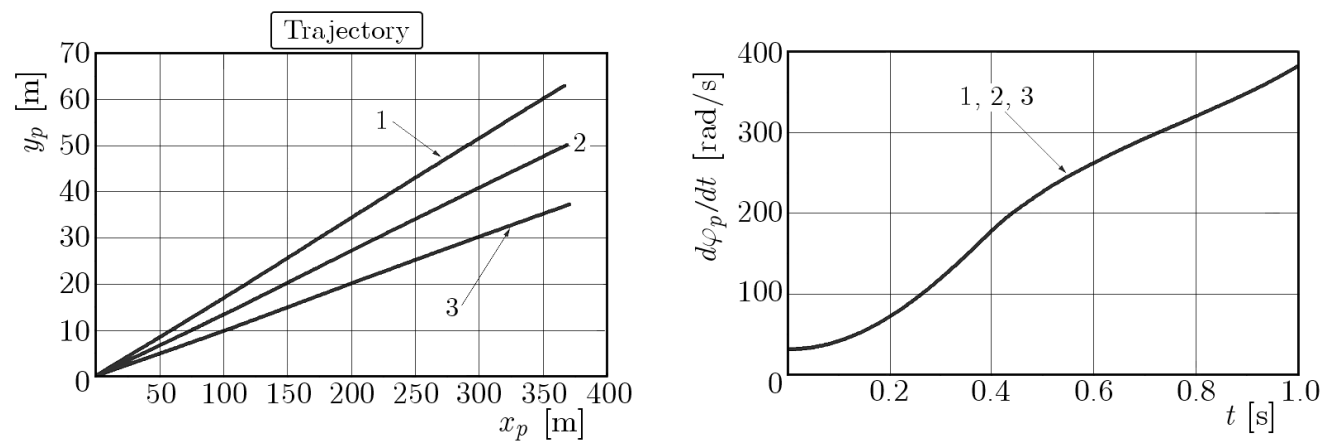

Fig. 11. Flight trajectory and angular velocity in the tilt motion of the rocket missile

on its direction. The results include three values of the initial angular velocity of the rocket in the inclination movement: $1-\dot{\theta}_{p}=0 \mathrm{rad} / \mathrm{s}, 4-\dot{\theta}_{p}=-0.2 \mathrm{rad} / \mathrm{s}, 5-\dot{\theta}_{p}=-0.4 \mathrm{rad} / \mathrm{s}$.

An insignificant change in the angular velocity of the rocket missile $\dot{\theta}_{p}$ at the moment when the missile leaves the launcher guide considerable changes the flight trajectory, see Fig. 12. Missiles 4 and 5 realize a wrong trajectory and consequently miss the target. Variability courses of the angular velocity in the tilt motion of rocket missiles 1,4 , and 5 are the same as in Fig. 12.
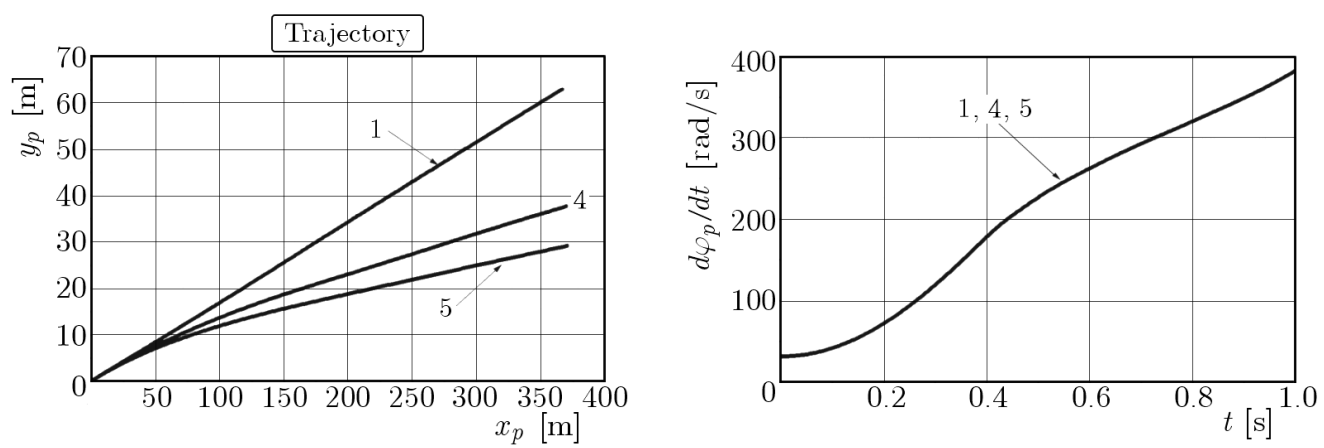

Fig. 12. Flight trajectory and angular velocity in the tilt motion of the rocket missile

\section{Conclusions}

An analysis of launching and flight of the unguided rocket missile NLPR-70 from the launcher WW-4 of the remotely controlled armament module ZSMU-70 has veen conducted as a part of the work. The launcher is positioned on a motor vehicle which is in the static equilibrium state at the moment the rocket motor is activated. The process of launching the missile from this armament module has been recorded with a high-speed camera at the military testing ground. The analysis of the recorded motion picture allowed us to evaluate the kinematic quantities characteristic for the launcher and missile performance.

A theoretical model of the launcher and missile motion has been developed after conducting the experimental studies and interpreting the results. The equations of the system motion representing the mathematical model have been derived on the basis of the physical model which has been formulated. The recorded motion of the armament module under field conditions indicated the possibility to design a virtual model with two dimensions. The theoretical model presented in the paper has been verified in experimental studies. Computer simulation and an analysis of the physical phenomena has been conducted taking into consideration analytical dependencies relating to the launcher motion and the unguided missile while launching. The inputs generated in the system while launching the missile have been determined as well as how they affect the launcher and the extent of the initial flight parameters. From the perspective of hitting the target of a certain surface, it is important for the initial missile flight parameters to allow that happen. The conducted considerations lead to the conclusion that the initial setting of the guide 
may differ explicitly from their values at the moment of launching the missile. For the unguided missiles it is specifically unfavourable as they cannot change their trajectory while flying. Hitting the target of a certain surface with the missile launched from the assembly under investigation is very problematic. The designed model allows for a comprehensive analysis of the launcher dynamics when launching the uncontrolled missile. It also enables evaluation of the initial flight parameters of the missile from the perspective of target reachability.

The paper presents a comparison of variability courses of selected physical quantities characterizing responses of the launcher to the missile launch in the case of active and passive mounting of the launcher platform. This allows us to evaluate the effectiveness of the implemented launcher stabilization system in form of two control systems operating independently of each other. The characteristic feature of the developed system is stabilization of each control system by only one point of attachment of the platform. The application of the system reducing vibrations of the platform determines the change of responses of the individual objects of the assembly to the occurring input. The vibrations of the launcher caused by the missile launch are reduced by the system stabilizing the platform. The passive mounting of the platform conveys disturbances and excites both the linear and angular vibrations of the launcher. The change of the passive mounting into active one significantly decreases the level of vibrations of the guide. It is important in the case of input occurring from the side where the gases are emitted by the rocket motor of the missile moving away from the launcher. Due to application of the active mounting, the input will not excite the guide to intensive vibrations. In the case of launching another missile, the reduced vibrations of the guide will not affect its launch in a negative way. The movement of the guide provides "comfort" for the missiles which are launched, and simultaneously improve the effectiveness in reaching the target. The stabilization system does not entirely eliminate the unfavourable phenomenon of rapid increase in the values of linear and angular acceleration of the platform the moment the missile leaves the guide, but reduces it.

It results from the research that the initial parameters of unguided missile flight affect its trajectory. The range of initial parameters of flight is determined by the dynamic characteristics of the launcher. The angle of setting the guide at the moment of launching the missile, but also its angular velocity in the inclination movement are extremely important. Both the value and the direction of the vector of the angular velocity are significant. Particularly adverse is the angular velocity resulting from the inclination of the rocket towards the Earth. The system of active stabilization of the launcher should be introduced in order to increase the effectiveness of the unguided rocket missile. The current structure of the remotely controlled module of the armament ZSMU-70 does not guarantee high effectiveness in the case of firing at a ground target whose overall dimensions are inconsiderable.

\section{References}

1. Biessonov A.P., 1967, Basis of the Dynamics of Mechanisms with a Variable Links Mass, Science, Moscow

2. De Silva C.W., 2007, Vibration Fundamentals and Practice, Taylor \& Francis Group, Boca Raton, London-New York

3. Dziopa Z., 2003, Vibration reduction of a close range missile launcher, Mechanics - Quarterly of the AGH University of Science and Technology, ISSN 0239-5282, 22, 3, 255-262

4. Dziopa Z., 2004, The dynamics of a rocket launcher placed on a self-propelled vehicle, Mechanical Engineering, Lviv, ISSN 1729-959, 3, 81, 23-30

5. Dziopa Z., 2006, An anti-aircraft self-propelled system as a system determining the initial parameters of the missile flight (in Polish), [In:] Mechanika w Lotnictwie, ML-XII 2006, J. Maryniak (Ed.), PTMTS Warsaw, ISBN 83-902194-6-8, 223-241 
6. Dziopa Z., 2007, Dynamics of a system of guides as an element of a launcher mounted on a motor vehicle, Scientific Notebooks of the Naval Academy, ISSN 0860-889X, XLVIII, 169K/1, 121-128

7. Dziopa Z., 2008, System stabilizing the launcher turret of the self-propelled anti-aircraft missile assembly, Magazine of Technology, Mechanics Notebook, ISSN 0011-4561, 101, 5, 31-41

8. Dziopa Z., Koruba Z., Krzysztofik I., 2010, Missile flight determined by the initial flight parameters of launching, 6th Conference on Avionics, Bezmiechowa 2010, ISBN 978-83-7199$-623-8$, p. 22

9. Engel Z., Kowal J., 1995, Controlling Vibro-Acoustic Processes, AGH Publishers, Cracow

10. Gacek J., Maryniak J., 1987, Modelling the dynamic properties of dynamic rotational bodies thrown from movable (in Polish), Biuletyn WAT, 10, 422, 81-96

11. Gantmacher F.R., 1970, Lectures in Analytical Mechanics, Translated from Russian by G. Yankovsky, Mir, Moscow

12. Inman D.J., 2006, Vibration with Control, John Wiley \& Sons, England

13. Osiecki J., 1994, Machine Dynamics (in Polish), Military University of Technology, Warsaw

14. Sibilski K., 2004, Modelling and Simulation of Dynamics of Flying Objects Motion, MH Printing House, Warsaw

15. SwietlickiJ W.A., 1963, Flying Objects Take-off Dynamics, Science, Moscow

16. ŻYLUK A., 2014, Numerical simulation of the effect of wind on the missile motion, Journal of Theoretical and Applied Mechanics, ISSN 1429-2955, 52, 2, 335-344

17. Żyluk A., Pietraszek M., 2014, Investigation of an additional oxidizer charge effect on selected operational characteristics of a solid-fuel rocket engine, Journal of Theoretical and Applied Mechanics, ISSN 1429-2955, 52, 1, 139-149 